\title{
Concepções de programas de prevenção à violência e promoção da cultura de paz
}

\author{
Concepts of violence prevention programs and promotion \\ of culture of peace
}

\author{
Stephanie Marques Moura Franco Belga, Kênia Lara Silva', \\ Roseni Rosângela de Sena ${ }^{1}$
}

\begin{abstract}
Resumo
Introdução: Este artigo propõe-se analisar programas de prevenção à violência com o objetivo de identificar as concepções que orientam o desenvolvimento desses programas e a relação com a promoção da cultura de paz. Método: Trata-se de um estudo de abordagem qualitativa aportada no referencial teórico-metodológico da análise de discurso crítica. Foram considerados para análise documentos online referentes a sete programas de prevenção da violência e promoção da cultura de paz desenvolvidos em Belo Horizonte - Minas Gerais. Resultados: A análise permitiu identificar uma cadeia de expressões nos objetivos dos programas que revelam aspectos ideológicos das políticas públicas cuja finalidade é a redução de incidências de eventos relacionados à violência. $\mathrm{O}$ discurso de promover uma cultura como mudança de paradigma e a fruição entre as pessoas aparece em alguns programas, no qual o fortalecimento de uma cultura de respeito aos direitos humanos e à cidadania está presente nas propostas. Em relação ao público-alvo dos programas, nota-se a focalização em segmentos caracterizados por faixas etárias mais jovens, com menor escolaridade. Conclusão: Embora haja uma relevância na implementação de programas de prevenção, ainda persistem desafios que Ihes são subjacentes aos aspectos ideológicos, políticos e práticos para construir a perspectiva da cultura de paz.

Palavras-chave: promoção da saúde; violência e políticas públicas.
\end{abstract}

\begin{abstract}
Introduction: This article aims to analyze violence prevention programs in order to identify the conceptions that guide their development and their relation with the promotion of culture of peace. Method:This is a qualitative study anchored in the theoretical and methodological reference of the critical discourse analysis. Online documents related to seven programs of violence prevention and promotion of culture of peace in Belo Horizonte - Minas Gerais, were considered for analysis. Results: The analysis allowed identifying a chain of expressions on the goals of the programs that reveal ideological aspects of public policies whose purpose is to reduce incidences of events related to violence. The speech of promoting culture as a paradigm shift and the enjoyment among people appears in some programs. Reinforcement of a culture of respect for human rights and citizenship is presented on the proposals. Regarding the target audience of the programs, there is a focus on segments characterized by younger age groups, lower level of education and residence in areas of high social vulnerability. Conclusion: Although the implementation of prevention programs is relevant, there are still underlying challenges to the ideological, political and practical perspective of building a culture of peace.
\end{abstract} Keywords: health promotion; violence and public policy. 


\section{INTRODUÇÃO}

No Brasil, desde meados da década de 1970, as estatísticas oficiais da violência apontam um aumento nas modalidades delituosas evidenciado por eventos por causas externas - homicídios, acidentes de transporte e suicídios ${ }^{1,2}$. O crescimento de eventos de violência tem se tornado um dos principais problemas de saúde pública e passou a dominar as preocupações de amplas camadas da população $0^{3,4}$.

A violência e seus efeitos perpassam a violação dos direitos humanos e sociais, o que significa agravo e ameaça à vida, às condições de trabalho, às relações interpessoais e à qualidade de vida. Isto implica pensar a violência não como um objeto próprio ao campo da saúde, mas considerando-a a complexidade de ser um fenômeno social e político 5 .

Diante desse contexto, é proeminente apontar a definição de políticas públicas que atuam na prevenção da violência. A resolução 49.25 da Organização Mundial de Saúde (OMS) propõe a prevenção da violência como uma prioridade no campo da saúde pública. Há também programas que atuam no controle de homicídios e prevenção de riscos e agravos, buscando atuar nos fatores desencadeantes da violência ${ }^{6,7}$. Entretanto, estudos mostram a aproximação de propostas sobre a violência restringindo seu combate e prevenção a uma mera questão de segurança pública e repressão policial, racionalizando ações em ferramentas de puniçãa ${ }^{8,9}$.

Em defesa de uma mudança de paradigma, espera-se que o desenvolvimento dos programas atue na perspectiva de que a violência não é um fenômeno estável, que se justifique por uma relação causal na qual um determinado fenômeno é consequência de outro, como pobreza gera violência, ou estratégias repressivas cerceará a violência ${ }^{8}$.

Nesse sentido, observa-se a necessidade de refletir sobre a aproximação da violência como um campo de conhecimento e intervenção social, considerando a amplitude dos aspectos correlacionados a esse fenômeno - individuais e estruturais, uma vez que é um processo que ocorre nas relações sociais modificando significativamente as formas de sociabilidade e o cotidiano das pessoas, manifestando-se tanto nos seus espaços sociais públicos e privados ${ }^{10}$ quanto em tempos de guerra ou de suposta paz.

A perspectiva de atuar sobre a violência não como um controle de fatores de risco avança para um processo de transformação de uma "[...] cultura de paz, em termos de valores, atitudes e comportamento individual e coletivo, bem como em termos de estruturas e funcionamentos institucionais" ${ }^{\text {"11 }}$ (p. 3). A Cultura de Paz acastelada pela Organização das Nações Unidas (ONU) propõe a transformação de valores, atitudes e comportamentos que sejam capazes de repensar a violência e a formação de conflitos $^{12}$.
Para abordar a violência, por meio de medidas de promoção da cultura de paz, torna-se necessário levar em consideração seus múltiplos níveis de determinação, macro ou micro estruturais e culturais. A cultura de paz propõe estimular e incentivar que a conquista da democracia seja construída mediante comunicação, diálogo, educação e respeito, resultando em uma resolução eficaz dos conflitos e construção da cidadania ${ }^{13}$. Desse modo, a cultura de paz defende ações orientadas pelos princípios: promoção dos direitos humanos e das liberdades fundamentais; promoção do desenvolvimento que respeite todas as formas de vida; promoção da educação para a paz; promoção da prática da não violência; promoção do fim da exclusão, injustiça e opressão político-econômica; e promoção da liberdade de expressão e diversidade cultural ${ }^{12}$.

Essas considerações apontam a necessidade de discutir entre os programas governamentais voltados para atuar sobre fenômeno da violência a incorporação da cultura de paz. Frente a isso, neste estudo propõe-se analisar programas de prevenção à violência com o objetivo de identificar as concepções que orientam o desenvolvimento desses programas e a relação com a promoção da cultura de paz.

\section{METODOLOGIA}

Trata-se de um estudo de abordagem qualitativa aportada no referencial teórico-metodológico da Análise Crítica de Discurso (ACD) proposta por Fairclough ${ }^{14,15}$. O autor propõe uma abordagem que articula as perspectivas social e linguística. O teórico utiliza o termo discurso para referir-se ao uso da linguagem como parte da prática social, um modo de ação e representação, social e historicamente situado, em uma relação dialética com a estrutura social. Propõe um quadro tridimensional de análise, considerando que cada evento discursivo tem as dimensões de texto, de prática discursiva e de prática social ${ }^{14}$.

Quanto a essas dimensões, a análise do texto será uma análise de forma e significado e será organizada nas categorias relacionadas a: vocabulário, gramática, coesão e estrutura textual ${ }^{14,15}$. $\mathrm{Na}$ análise da prática discursiva serão utilizadas categorias relacionadas à produção, distribuição e consumo do texto $^{14}$. Por sua vez, a prática social corresponde a uma forma de atividade social relativamente estável, que inclui diversos elementos sociais relacionados dialeticamente com atividades, sujeitos e suas relações sociais, instrumentos, objetos, tempo e lugar, formas de consciência, valores e discurso ${ }^{14,15}$.

A análise a ser realizada neste artigo busca compreender como essas construções discursivas se inserem nas concepções dos programas, sendo marcadas e delimitadas por elementos da formulação das políticas públicas, que conferem maior ou menor magnitude ou legitimidade a essas construções.

Para compor o corpus analisado, foi realizado o mapeamento dos programas que têm o tema promoção da cultura de paz ou 
prevenção da violência em Belo Horizonte, Minas Gerais/Brasil. A identificação dos programas foi realizada por meio de levantamento de fontes secundárias (análise documental; web site). A análise dos dados foi orientada pelas concepções dos programas identificando o público-alvo; objetivos; abordagem central dos programas; temáticas discutidas; abordagens ou metodologias empregadas; ocorrência de parcerias e intersetorialidade. Ao final do mapeamento, obteve-se o corpus de sete programas de prevenção à violência e promoção da cultura de paz (Quadro 1).

\section{RESULTADOS E DISCUSSÃO}

A partir da análise dos programas de prevenção da violência e promoção da cultura de paz, foi possível identificar aspectos que definem estrutural e conjunturalmente os programas no campo das ações.

Identificam-se os aspectos ideológicos das políticas públicas nas quais uma cadeia de expressões textuais é reiterada nos objetivos dos programas na tentativa de defender, ou justificar, um conjunto de benefícios que serão advindos da prática. Reduzir incidências de eventos relacionados à criminalidade e a prevenção da violência (P1); reduzir morbimortalidade em decorrência de eventos violentos (P3); e dotar comportamentos em jovens para viver sem drogas e violência (P4) desempenham significações a partir de um controle macrossocial que atribui metas e estabelece padrões normativos para moldar comportamentos dentro da sociedade.
Diversos estudos têm demonstrado programas centrados na construção de normas de convivência e respeito, como modos de prevenção da violência e do crime ${ }^{16,17}$. Sabe-se que há violências ocasionais, outras que são permanentes, e elas dependem de ações que considerem as multideterminações do fenômeno. Por isso, a prevenção da violência não pode ser feita de maneira determinista, mas sim com estratégias que modifiquem as relações sociais.

A definição do público-alvo delimita o campo de atuação dos programas. Nesse aspecto, nos programas analisados, sobressai a abrangência de segmentos da população caracterizados por faixas etárias mais jovens, com menor escolaridade e marcados pela focalização territorial em áreas de alta vulnerabilidade social. A definição deste público é um fator determinante para a condução do programa, e pode ser justificado por estar entre os jovens a maior incidência de acidentes de trânsito e homicídio ${ }^{18}$. Além disso, os jovens são aqueles que atendem por um tipo ideal de risco: jovens negros e/ou mulatos do sexo masculino, oriundos das classes vulnerabilizadas ${ }^{8}$.

Desse modo, há uma diferença social nos programas que direciona o desenvolvimento das práticas marcado pela desigualdade social e distribuição desigual na sociedade. Do ponto de vista sociológico, a desigualdade social supõe o acesso diferenciado a bens, construído e definido socialmente, através de uma dada organização de posições no interior do sistema social ${ }^{19}$. No entanto, há um tensionamento para se promover a cultura de paz em sociedades marcadas pela desigualdade social.

Quadro 1. Características dos programas de prevenção da violência e promoção da cultura de paz

\begin{tabular}{|c|c|c|c|c|}
\hline Programa & Objetivo & Público-alvo & Intersetorialidade & $\begin{array}{c}\text { Metodologia/ } \\
\text { abordagem }\end{array}$ \\
\hline P1 & $\begin{array}{l}\text { Reduzir a incidência de homicídios dolosos } \\
\text { mediante prevenção nas áreas de risco, cujo } \\
\text { indicadores de criminalidade violenta o } \\
\text { justifiquem }\end{array}$ & $\begin{array}{l}\text { Moradores de áreas de } \\
\text { risco, jovens de } 12 \text { a } 24 \\
\text { anos }\end{array}$ & Sim & $\begin{array}{l}\text { Proteção social, } \\
\text { intervenções } \\
\text { estratégicas, oficinas. }\end{array}$ \\
\hline $\mathrm{P} 2$ & $\begin{array}{l}\text { Potencializar a participação cidadã de } \\
\text { determinados segmentos sociais e também } \\
\text { estimular as interfaces entre os diferentes } \\
\text { atores sociais comunitários locais }\end{array}$ & $\begin{array}{l}\text { Moradores de áreas de } \\
\text { risco, faixa etária livre }\end{array}$ & Não & $\begin{array}{l}\text { Orientação, práticas de } \\
\text { resolução de conflitos. }\end{array}$ \\
\hline P3 & $\begin{array}{l}\text { Reduzir a morbimortalidade e os impactos } \\
\text { psicológicos, sociais e culturais da violência }\end{array}$ & Gestores intrassetoriais & Não & Caráter consultivo \\
\hline $\mathrm{P} 4$ & $\begin{array}{l}\text { Dotar jovens estudantes de informações } \\
\text { e habilidades necessárias para viver de } \\
\text { maneira saudável, sem drogas e violência }\end{array}$ & $\begin{array}{l}\text { Alunos, professores e } \\
\text { familiares das escolas } \\
\text { participantes do } \\
\text { programa }\end{array}$ & Sim & Palestras \\
\hline P5 & $\begin{array}{l}\text { Promover a formação e a capacitação dos } \\
\text { educadores }\end{array}$ & $\begin{array}{l}\text { Educadores da rede } \\
\text { pública }\end{array}$ & Não & Capacitação \\
\hline P6 & $\begin{array}{l}\text { Subsidiar a escola sobre } \\
\text { conceitos referentes à temática da prevenção } \\
\text { da violência }\end{array}$ & $\begin{array}{l}\text { Alunos, professores e } \\
\text { familiares das escolas } \\
\text { participantes do } \\
\text { programa }\end{array}$ & Sim & Palestras e capacitações \\
\hline P7 & $\begin{array}{l}\text { Formação e fortalecimento sobre cultura de } \\
\text { respeito aos direitos humanos e cidadania }\end{array}$ & $\begin{array}{l}\text { Moradores de áreas de } \\
\text { risco, faixa etária adulta }\end{array}$ & Não & $\begin{array}{l}\text { Orientações e ações } \\
\text { educativas }\end{array}$ \\
\hline
\end{tabular}

Fonte: Elaborado pelos autores 
A incorporação ideológica da promoção da cultura de paz nos programas pode se representar por duas formas. Primeiro, o fato de focalizar as práticas em públicos específicos referente à necessidade de "[...] manutenção e obtenção dos direitos aos cidadãos, uma vez que esses direitos ainda não estão em vigor para todas as classes sociais e etnias" 20 (p. 148). O segundo fato relaciona-se à possibilidade de "[...] reinstalar processos de violência em áreas de periferias que se caracterizam como um território estigmatizado que apenas produz subjetividades atreladas a um contexto social violento"20 (p. 142).

Assim, conjecturando esses elementos com a estrutura social, nota-se que o corpus analisado apresenta um contexto implícito na dimensão da prática discursiva. Analisando-se os programas de prevenção da violência, promoção da cultura de paz, o contexto da criação desses programas está relacionado ao processo histórico da violência na sociedade. Os índices de criminalidade no Brasil, a falta de oportunidades das pessoas menos favorecidas, a violência policial, impulsionam a formulação de políticas nesse campo ${ }^{21}$.

A prática discursiva reproduz aspectos da estrutura social, como elementos de controle e desigualdade social, que são notórios na abordagem das práticas, na definição do público-alvo, na composição do projeto dos programas e na localização das práticas que estão predominantemente em áreas de risco. Essa construção discursiva dos programas legitima conceitos e ideologias de uma luta de classes entre o público que compõe as práticas e os aspectos determinantes na formulação dos programas.

Quanto à abordagem utilizada, destaca-se em alguns programas o caráter repressivo, que se orienta para a transformação de comportamento baseada na autoridade. No programa P4, o discurso baseia-se na concepção de uma educação repressiva, traduzida em uma prática social orientada na não distinção do uso e abuso de drogas associado à dependência. Essa concepção denota uma abordagem autoritária e moralista com relação ao fenômeno ${ }^{21}$. Nos programas P1, P4 e P6, algumas abordagens utilizadas centram-se no controle da delinquência e violência, restringindo a prevenção a aspectos de segurança pública e repressão policial. A esse aspecto, o fim da violência não deve se limitar a ações punitivas e repressivas ${ }^{8}$.

Todavia, foram analisados outros tipos de abordagens nos programas. Verificam-se nos discursos algumas propostas de oficinas artísticas e de valorização de elementos culturais (P1) que propõem, em seu desenvolvimento, garantir a participação social e reflexão de temas que abarcam os direitos humanos (P1, P5, P7). Além disso, foram analisados em todos os programas elementos que indicam o uso de estratégias de sensibilização e conceituação do fenômeno da violência, funcionando por meio de palestras, capacitações e encaminhamentos a órgãos competentes.
Contudo, a maioria dos programas organiza-se por meio de intervenções pontuais e de sessão única que utilizam recursos metodológicos típicos para apresentação teórica e de conteúdos (P2, P4, P6, P7). O desenvolvimento de propostas pontuais indica uma lacuna na possibilidade de se pensar uma prática com caráter de mudança social, visto que na intervenção pontual o alcance torna-se limitado diante dos desafios de uma proposta que requer uma ação permanente de criação e transformação da realidade.

Propostas que incentivam a participação social vão ao encontro da cultura de paz, pois operam com princípios democráticos e novas formas de solidariedade e transformação social, implicando a melhoria nas relações sociais, a participação ativa e o empoderamento das pessoas ${ }^{22}$. Potencializar a participação cidadã de determinados segmentos sociais é apontado como objetivo central em cinco programas (P1, P2, P5, P6, P7).

Outra característica analisada é o aspecto da intersetorialidade nos programas. A intersetorialidade presente em três programas (P1, P4, P6) sinaliza um consenso discursivo sobre a potencialidade da articulação como dispositivo de construção compartilhada de diferentes setores com vistas a intervenções estratégicas, para superar as hierarquias institucionais e as relações de poder entre setores, políticas e segmentos sociais ${ }^{23}$.

Quanto às concepções de cultura de paz, os discursos revelam elementos que se aproximam da perspectiva defendida pela ONU (Quadro 2) com expressão dos princípios de: promoção da prática à não violência; promoção dos direitos humanos e das liberdades fundamentais; e promoção do fim da exclusão, injustiça e opressão político-econômica.

O princípio Promoção da prática à não violência está presente em seis programas. $O$ fato desta concepção estar presente na maioria dos programas pode estar relacionado a uma aproximação conceitual entre a construção social do discurso da não violência e da prevenção da violência, sendo por vezes entendidos como sinônimos. Nessa perspectiva, consideram-se os enunciados pautados na reflexão da prevenção da violência (P1, P3, P4, P5), ações de vigilância e monitoramento de índices de violência (P1, P3, P6), desenvolvimento de habilidades necessárias para viver de maneira saudável, sem drogas e violência entre o público jovem $(\mathrm{P} 4, \mathrm{P} 6)$.

O princípio Promoção dos direitos humanos e das liberdades fundamentais é citado em cinco programas, tanto na perspectiva de reflexão sobre temas relacionados à cidadania e aos direitos humanos (P1, P2, P5, P6, P7) quanto no estímulo ao direito de ir e vir (P1). Nesse sentido, o incentivo de práticas traduz discursos em defesa da democracia que leva em conta os conflitos cotidianos decorrentes da intolerância à diferença e das condições desiguais de existência, sejam elas subjetivas ou objetivas. 
Quadro 2. Concepções de cultura de paz que orientam o desenvolvimento dos programas

\begin{tabular}{|c|c|c|c|c|c|c|c|}
\hline $\begin{array}{c}\text { Princípios de } \\
\text { Cultura de Paz }\end{array}$ & P1 & P2 & P3 & P4 & P5 & P6 & P7 \\
\hline $\begin{array}{l}\text { Promoção } \\
\text { dos direitos } \\
\text { humanos e } \\
\text { das liberdades } \\
\text { fundamentais }\end{array}$ & $\begin{array}{l}\text { Promoção } \\
\text { dos direitos } \\
\text { humanos e } \\
\text { cidadania; } \\
\text { potencializar } \\
\text { o direito de ir } \\
\text { e vir }\end{array}$ & $\begin{array}{l}\text { Promoção } \\
\text { dos direitos } \\
\text { humanos e } \\
\text { cidadania }\end{array}$ & & & $\begin{array}{l}\text { Educação } \\
\text { em direitos } \\
\text { humanos e } \\
\text { cidadania }\end{array}$ & & $\begin{array}{l}\text { Formação e } \\
\text { fortalecimento } \\
\text { dos direitos } \\
\text { humanos }\end{array}$ \\
\hline $\begin{array}{l}\text { Promoção do } \\
\text { desenvolvimento } \\
\text { que respeite } \\
\text { todas as formas } \\
\text { de vida }\end{array}$ & & $\begin{array}{l}\text { Participação } \\
\text { cidadã de } \\
\text { determinados } \\
\text { segmentos } \\
\text { sociais } \\
\end{array}$ & & & & $\begin{array}{l}\text { Capacitação } \\
\text { em relação a } \\
\text { intolerâncias } \\
\text { diferenças }\end{array}$ & $\begin{array}{l}\text { Enfoque nos } \\
\text { segmentos } \\
\text { sociais } \\
\text { vulnerabilizados }\end{array}$ \\
\hline $\begin{array}{l}\text { Promoção da } \\
\text { educação para } \\
\text { a paz }\end{array}$ & & & & & $\begin{array}{l}\text { Capacitação } \\
\text { vinculando-a } \\
\text { aos conceitos } \\
\text { de diálogo, } \\
\text { interação e } \\
\text { articulação }\end{array}$ & $\begin{array}{l}\text { Ações de } \\
\text { fomento da paz } \\
\text { entre as pessoas }\end{array}$ & \\
\hline $\begin{array}{l}\text { Promoção da } \\
\text { prática à não } \\
\text { violência }\end{array}$ & $\begin{array}{l}\text { Prevenção } \\
\text { da violência; } \\
\text { monitoramento } \\
\text { da } \\
\text { criminalidade }\end{array}$ & $\begin{array}{l}\text { Resolução de } \\
\text { conflitos }\end{array}$ & $\begin{array}{l}\text { Vigilância e } \\
\text { prevenção da } \\
\text { violência }\end{array}$ & $\begin{array}{l}\text { Desenvolver } \\
\text { habilidades } \\
\text { para viver } \\
\text { sem drogas e } \\
\text { violência }\end{array}$ & $\begin{array}{l}\text { Desenvolver } \\
\text { material } \\
\text { didático que } \\
\text { estimule a } \\
\text { prevenção da } \\
\text { violência }\end{array}$ & $\begin{array}{l}\text { Prevenção da } \\
\text { violência }\end{array}$ & \\
\hline $\begin{array}{l}\text { Promoção do } \\
\text { fim da exclusão, } \\
\text { injustiça e } \\
\text { opressão } \\
\text { político- } \\
\text { econômica }\end{array}$ & $\begin{array}{l}\text { Intervenção } \\
\text { estratégica à } \\
\text { justiça social }\end{array}$ & $\begin{array}{l}\text { Acesso à justiça } \\
\text { social }\end{array}$ & $\begin{array}{l}\text { Reduzir } \\
\text { impactos } \\
\text { psicológicos, } \\
\text { sociais e } \\
\text { culturais }\end{array}$ & & $\begin{array}{l}\text { Justiça na } \\
\text { escola }\end{array}$ & & $\begin{array}{l}\text { Acesso à } \\
\text { justiça social; } \\
\text { Divulgação dos } \\
\text { direitos }\end{array}$ \\
\hline $\begin{array}{l}\text { Promoção da } \\
\text { liberdade de } \\
\text { expressão e } \\
\text { diversidade } \\
\text { cultural } \\
\end{array}$ & $\begin{array}{l}\text { Oficinas } \\
\text { artísticas, } \\
\text { valorização } \\
\text { de elementos } \\
\text { culturais }\end{array}$ & & & & & & \\
\hline
\end{tabular}

Fonte: Elaborado pelos autores

A Promoção do fim da exclusão, injustiça e opressão político-econômica apresenta-se registrada em cinco programas, centrando-se nos elementos da exclusão social (P5) e injustiça na perspectiva de acesso à justiça social e a fruição dos direitos da população (P1, P2, P4, P7). O discurso de justiça pressupõe ideias de equidade na divisão social e do trabalho, considerando as necessidades individuais para buscar soluções justas para todos. Desse modo, consideram-se esses aspectos como expressões da ação coletiva que remete ao interesse dos indivíduos pela promoção de bens públicos e bem-estar ${ }^{24}$.

Ainda na análise dos programas, outros aspectos apresentados relacionam-se ao respeito à diversidade (P2, P6, P7) e a promoção da diversidade cultural (P1). Pode-se afirmar que tais concepções avançam em novos modos de pensar o enfrentamento da violência, uma vez que o compartilhamento da cultura coletiva, entre eles normas, regras e valores sociais distintos, acabam por conduzir a novas práticas sociais.

A análise permite inferir que os programas estão intrinsecamente relacionados à prevenção e à resolução não violenta de conflitos. A construção da cultura de paz se demonstrou presente em alguns discursos que orientam a formulação dos programas, avançando para um sentido positivo, que não pode ser definida pela simples ausência ou prevenção da violência, mas consolidada na ideia de que as interações humanas podem ser geradoras da paz e desenvolvidas a partir de práticas proativas que dependem dos sujeitos envolvidos no processo ${ }^{24}$.

\section{CONCLUSÃO}

Embora haja uma relevância na implementação desses programas de prevenção, ainda persistem lacunas quanto ao avanço em uma perspectiva de adotar à cultura de paz para 
o enfrentamento da violência. Práticas que fortaleçam uma cultura de respeito aos direitos humanos e às diversidades, $\mathrm{e}$ que operem na mudança de paradigmas da violência, avançam na conquista da democracia, na resolução eficaz dos conflitos e na construção da cidadania.

Destaca-se nos programas analisados o jovem como alvo. Mas ainda são incipientes nos discursos ações que o tornam protagonista, pois não basta desenvolver ações com intuito de estabelecer comportamentos ideais que enfoquem na redução dos diversos comportamentos de risco dos jovens, tais como o consumo de drogas e a prática violenta. Neste âmbito, os jovens devem ser entendidos como agentes ativos que promovam mudanças capazes de interiorizar novos modos de viver e desconstruir paradigmas.

Esse artigo procurou demonstrar que há ainda muito a ser investigado quanto aos avanços na prática dos programas de prevenção da violência. Os resultados do estudo servem também como um convite para outras pesquisas: a construção dos discursos dos jovens quanto aos programas e a análise desses programas na mudança social, já que os discursos utilizados demonstraram potencial para atuar na promoção social dos indivíduos.

\section{REFERÊNCIAS}

1. Mascarenhas MDM, Neves ACM, Monteiro RA, Silva MMA, Malta DC. Atendimentos de emergência por causas externas e consumo de bebida alcoólica - Capitais e Distrito Federal, Brasil, 2011. Cien Saude Colet. 2015;20(4):1037-46. PMid:25923616. http://dx.doi.org/10.1590/141381232015204.14842014 .

2. Pan American Health Organization. Prevention of alcohol-related injuries in the Americas: from evidence to policy action. Washington: PAHO; 2013.

3. Silva LAM. Violência urbana", segurança pública e favelas: o caso do Rio de Janeiro atual. Cad CRH. 2010;23(59):283-300. http://dx.doi.org/10.1590/ S0103-49792010000200006.

4. Fraade-Blanar L, Concha-Eastman A, Baker T. Injury in the Americas: the relative burden and chalenge. Rev Panam Salud Publica. 2007;22(4):254-9. PMid:18078587. http://dx.doi.org/10.1590/S1020-49892007000900005.

5. Minayo MC, Souza ER.É possível prevenir a violência? Reflexões a partir do campo da saúde pública. Cien Saude Colet. 1999;4(1):7-23. http://dx.doi. org/10.1590/S1413-81231999000100002.

6. Baeto FCC, Silva BFA, Tavares R. Crime e estratégias de policiamento. Dados. 2008;51(3):601-31.

7. Farrington DP. Fatores de risco para a violência juvenil. In: Debarbieux E, Blaya C, editores. Violência nas escolas e políticas públicas. Brasília: UNESCO; 2002

8. Cruz No O, Moreira MR. A concretização de políticas públicas em direção à prevenção da violência estrutural. Cien Saude Colet. 1999;4(1):33-52. http://dx.doi.org/10.1590/S1413-81231999000100004.

9. Sapori LF. Segurança pública no Brasil: desafios e perspectivas. Rio de Janeiro: Editora FGV; 2007.

10. Baierl LF. Medo social: dilemas cotidianos. Ponto-e-Vírgula. 2008;3:138-51.

11. Organização das Nações Unidas para a Educação, a Ciência e a Cultura. Cultura de paz: da reflexão à ação: balanço da Década Internacional da Promoção da Cultura de Paz e Não Violência em Benefício das Crianças do Mundo. Brasília: UNESCO; 2010.

12. Organização das Nações Unidas. Declaração e Programa de Ação sobre uma Cultura de Paz. Resolução aprovada por Assembléia Geral em 06 de outubro de 1999, nº 53/243. New York: ONU; 1999. Original: Declaración y Programa de Acción sobre uma Cultura de Paz.

13. Ruíz CA. Teología y cultura de paz. Cultura de Paz. 2014;20(62):16-24.
14. Fairclough N. Discurso e mudança social. 2. ed. Brasília: Editora Universidade de Brasília; 2008. p. 89-116.

15. Fairclough N, Chouliaraki L. Social life and critical social science. In: Fairclough N, Chouliaraki L. Discourse in late modernity: rethinking critical discourse analysis. Edinburgh: Edinburgh University Press; 2001. cap. 2, p. 19-36.

16. Tremblay RE, Mâsse LC, Pagani L, Vitaro F. From childhood physical aggression to adolescent maladjustment: the montreal prevention experiment. In: Peters RD, McMahon RJ, editores. Preventing childhood disorders, substance abuse, and delinquency. Thousand Oaks: Sage; 1996. (Banff International Behavioral Science Series, 3).

17. Chaux E. Buscando pistas para prevenir la violência urbana en Colombia: conflictos y agresión entre niños y adolescentes de Bogotá. Rev Estud Soc. 2002;12:41-51.

18. Burrone MS, Bella M, Acosta L, Villace B, López de Neira MJ, Fernández $\mathrm{R}$, et al. Estudio de muertes por causas violentas: un análisis de tendencia en jóvenes, Argentina, 2000-2008. Cad Saude Colet. 2012;20(4):460-5. http://dx.doi.org/10.1590/S1414-462X2012000400009.

19. Sorj B. Uma nota sobre os estudos de desigualdade social no Brasil. In: Anais do $19^{\circ}$ Encontro Anual da ANPOCS; 2001; Caxambu. São Paulo: ANPOCS; 2001.

20. Ferreira AL, Silveira MFG, Peixoto ACL. Promoção de cultura de paz e resiliência: um estudo de caso do projeto de extensão rede coque vive da UFPE. Rev Reflexão \& Ação. 2013;21(1):140-68.

21. Paes VGF, Ribeiro LML. Produção acadêmica sobre práticas de segurança pública e justiça criminal. Confluências. 2014;16(3):9-33.

22. Almeida HN, Albuquerque CP, Santos CC. Culture of peace and social mediation. Foundations for building more just and participatory society. Mediaciones Sociales. 2013;12:132-57.

23. Silva KL, Sena RR, Akerman M, Belga SMM, Rodrigues AT. Intersetorialidade, determinantes socioambientais e promoção da saúde. Cien Saude Colet. 2014;19(11):4361-70. http://dx.doi.org/10.1590/1413-812320141911.10042014.

24. Moreira LS, Branco AU. Cultura de paz, moralidade e virtudes cívicas: contribuições da psicologia cultural. Psicol. Argum. 2012;30(68):161-70. http://dx.doi.org/10.7213/psicol.argum.5894.

Recebido em: Mar. 14, 2016 Aprovado em: Maio 02, 2017 